\title{
Neural responses to threat and reward interact to predict stress-related problem drinking: A novel protective role of the amygdala
}

\author{
Yuliya S Nikolova* and Ahmad R Hariri
}

\begin{abstract}
Background: Research into neural mechanisms of drug abuse risk has focused on the role of dysfunction in neural circuits for reward. In contrast, few studies have examined the role of dysfunction in neural circuits of threat in mediating drug abuse risk. Although typically regarded as a risk factor for mood and anxiety disorders, threat-related amygdala reactivity may serve as a protective factor against substance use disorders, particularly in individuals with exaggerated responsiveness to reward.

Findings: We used well-established neuroimaging paradigms to probe threat-related amygdala and reward-related ventral striatum reactivity in a sample of 200 young adult students from the ongoing Duke Neurogenetics Study. Recent life stress and problem drinking were assessed using self-report. We found a significant three-way interaction between threat-related amygdala reactivity, reward-related ventral striatum reactivity, and recent stress, wherein individuals with higher reward-related ventral striatum reactivity exhibit higher levels of problem drinking in the context of stress, but only if they also have lower threat-related amygdala reactivity. This three-way interaction predicted both contemporaneous problem drinking and problem drinking reported three-months later in a subset of participants.
\end{abstract}

Conclusions: These findings suggest complex interactions between stress and neural responsiveness to both threat and reward mediate problem drinking. Furthermore, they highlight a novel protective role for threat-related amygdala reactivity against drug use in individuals with high neural reactivity to reward.

Keywords: Amygdala, Ventral striatum, Threat, Reward, Stress, Alcohol

\section{Findings}

Increased amygdala reactivity to threat has been consistently associated with heightened risk for mood and anxiety disorders [1]. In contrast to this heightened risk, a few studies have suggested that threat-related amygdala reactivity may buffer risk for drug abuse. Specifically, one study reported that individuals at high familial risk for alcoholism exhibit relatively reduced threat-related amygdala reactivity [2]. The authors speculate that this pattern may indicate reduced sensitivity to the harmful consequences of excessive alcohol use in those at risk.

Consistent with these findings, a recent study has linked a genetic variant conferring increased risk for drug

\footnotetext{
*Correspondence: yuliya.nikolova@duke.edu

Laboratory of NeuroGenetics, Department of Psychology \& Neuroscience and Institute for Genome Sciences \& Policy, Duke University, NC 27708 Durham,
} USA

\section{() Biomed Central}

abuse [3] with relatively decreased threat-related amygdala reactivity [4]. Interestingly, the same genetic risk variant was associated with heightened reward-related reactivity of the ventral striatum (VS), a neural phenotype associated with both risk for and pathophysiology of drug abuse [5,6]. These data suggest a potentially synergistic effect of threat-related amygdala reactivity and reward-related VS reactivity in precipitating drug abuse risk. In addition to variability in these neural phenotypes, drug abuse risk is moderated by environmental factors, such as recent life stress [7]. Both VS and amygdala function are also affected by stress [8], suggesting that complex interactions between these neural circuits may contribute to variability in stress-related risk for drug abuse.

Here, we explore the interactions of recent life stress, threat-related amygdala and reward-related VS reactivity 
in predicting variability in self-reported problem drinking in a sample of 200 young adults. We focused on drinking because alcohol is the most commonly used and abused drug in adolescents and young adults [9], and its use is often triggered by stress [7]. Using two well-characterized BOLD fMRI paradigms ([10] Figure 1A-B), we quantified threat-related amygdala and reward-related VS reactivity. Recent life stress and problem drinking were assessed using the Life Events Scale for Students (LESS, [11]) and the Alcohol Use Disorder Identification Test (AUDIT, [12]), respectively. Based on prior research, we predicted that higher threat-related amygdala reactivity would protect against increased problem drinking in the context of stress, particularly in those whose risk is exaggerated by higher reward-related VS reactivity.

As expected [7], there was a significant positive correlation between recent stress and problem drinking $(r=0.22$, $\mathrm{p}=0.004$ ). Critically, however, this relationship was moderated by amygdala and VS reactivity (Figure 1A-B). Specifically, a three-way interaction predicting problem drinking emerged between recent stress, left amygdala reactivity, and left VS reactivity $\left(\Delta R^{2}=0.035, b=-0.26\right.$, $\mathrm{p}=0.012$ ). Among participants with low VS reactivity (1 SD below mean; Figure 1C), stress did not predict any increases in drinking, regardless of amygdala reactivity.
Among participants with high VS reactivity (1 SD above mean), who are likely to be at increased risk for drug abuse [5], stress predicted increased problem drinking only for those who also had low amygdala reactivity (1 SD below mean; Figure 1D). This three-way interaction remained significant after controlling for gender, age, and race/ethnicity $\left(\Delta \mathrm{R}^{2}=0.031, \mathrm{~b}=-0.25, \mathrm{p}=0.012\right)$. There was no such interaction for right VS or amygdala reactivity ( $\mathrm{p}$ values $>0.10$ ), and no significant main effects of either amygdala or VS reactivity on problem drinking ( $\mathrm{p}$ values $>0.14$ ).

Demonstrating the specificity of these findings to recent, as opposed to early, life stress, the three-way interaction remained significant when total scores from the Childhood Trauma Questionnaire [13] were added as an additional covariate (left VS: $\mathrm{R}^{2}=0.033, \mathrm{~b}=-0.25$, $\mathrm{p}=0.011$ ). Furthermore, childhood trauma did not interact with amygdala or VS reactivity to predict problem drinking ( $p$ values $>0.63$ ). Finally, the same three-way interaction emerged in a subsample of participants $(\mathrm{N}=85)$ who completed a three-month follow-up assessment of stress and problem drinking (without covariates: $\Delta \mathrm{R}^{2}=0.085, \mathrm{~b}=-0.365, \mathrm{p}=0.008$; with covariates: $\left.\Delta \mathrm{R}^{2}=0.063, \mathrm{~b}=-0.324, \mathrm{p}=0.019\right)$. The temporal stability of this interaction suggests that stress-related problem

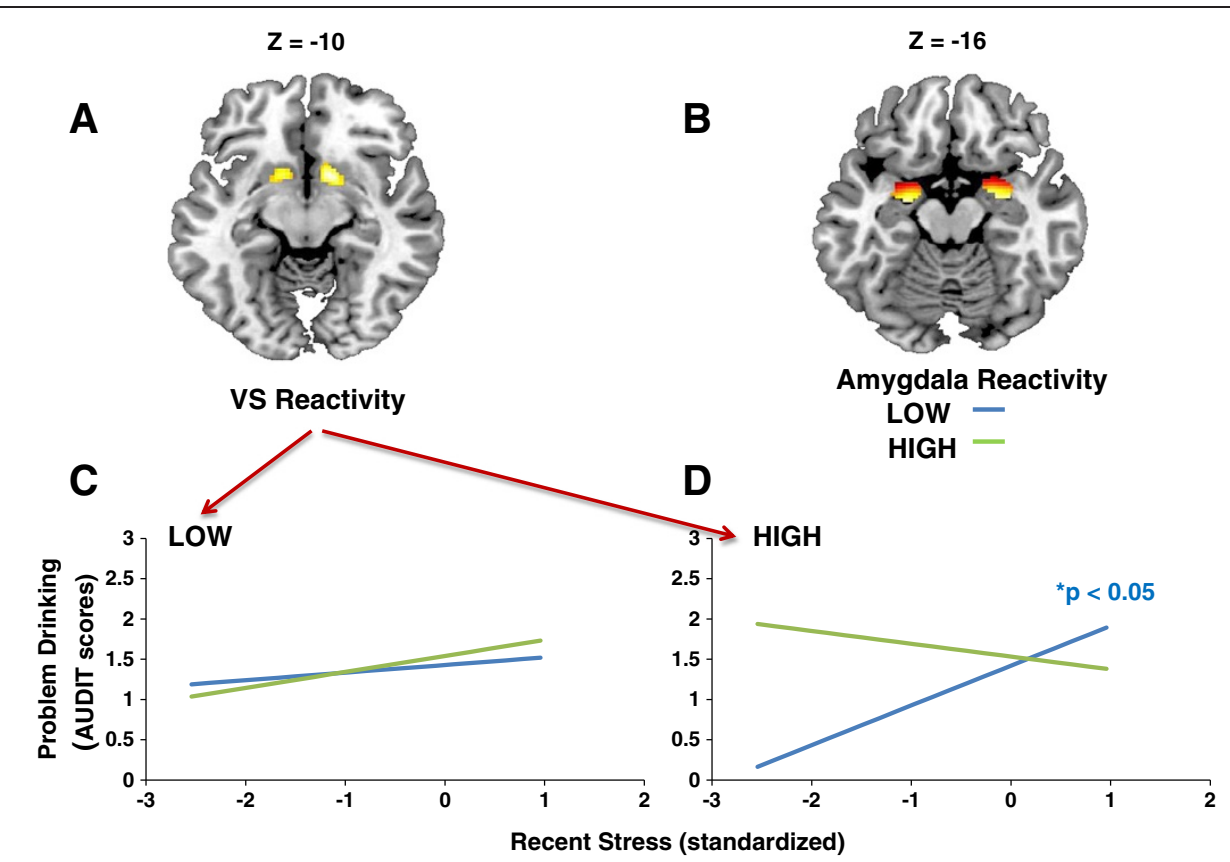

Figure 1 Amygdala and VS reactivity interact with recent stress to predict problem drinking. (A) Statistical parametric map illustrating mean bilateral threat-related amygdala reactivity (left: $x=-22, y=-6, z=-18, t=19.76, p<0.000001, k_{E}=173 ;$ right: $x=28, y=-4, z=-20, t=20.16$, $p<0.000001, k_{E}=199$ ). (B) Statistical parametric map illustrating mean bilateral reward-related VS reactivity (left: $x=-12, y=10, z=-10, t=6.19, p=3.07 \times 10^{-7}$, $k_{E}=357$; right: $x=12, y=10, z=-8, t=7.31, p=1.03 \times 10^{-9}, k_{E}=383$ ). Activation clusters in (A) and (B) are overlaid onto canonical structural brain images in the axial plane. (C) Among participants with low VS reactivity, (1 SD below the mean), recent stress (LESS Highest Impact) was not associated with increased problem drinking (total scores on the AUDIT; square root transformed) regardless of amygdala reactivity. (D) For participants with high (1 SD above the mean) VS reactivity, recent stress predicted significant increases in problem drinking only for those who also had relatively low (1 SD below the mean) amygdala reactivity (blue line). Plotted values are adjusted for sex, age and race/ethnicity. 
drinking reflects rather than affects the relative neural responsiveness to threat and reward.

An important caveat to consider when interpreting these findings is the possibility that participants drinking more alcohol may experience more stressful life events partially as a result of their increased drinking, rather than the other way around. Since our measures of stress and problem drinking are based on retrospective selfreport spanning the past 12 months, the directionality of the association between stress and drinking cannot be determined on the basis of these analyses. Thus we cannot rule out the alternative interpretation that individuals with high VS reactivity and low amygdala reactivity are more likely to experience highly impactful stressful life events in the context of problem drinking. This interpretation would be consistent with a heightened drive to pursue immediate rewards, coupled with a reduced ability to recognize and avoid threat in those individuals.

Limitations notwithstanding, we provide novel evidence that recent life stress is associated with increased problem drinking only in individuals with higher rewardrelated VS reactivity and lower threat-related amygdala reactivity. Consistent with the relative temporal stability of amygdala [14] and VS [15] reactivity, the interactions between these neural phenotypes and recent life stress predicted future problem drinking in a subset of participants. This finding suggests that the pattern we observe spans longer periods of time and may be useful in identifying individuals at particularly high risk for developing alcohol and possibly other substance use disorders in the wake of stress. Future research identifying factors that predict the observed variability in neural responsiveness to threat and reward (e.g., functional genetic polymorphisms) can inform the development of biomarkers for drug abuse risk and interventions targeting these specific intermediate phenotypes.

\section{Abbreviations}

AUDIT: Alcohol Use Disorder Identification Test; BOLD fMRI: Blood oxygen level dependent functional magnetic resonance imaging; LESS: Life Events Scale for Students; Vs: Ventral striatum.

\section{Competing interests}

YSN and ARH declare no competing interests.

\section{Authors' contributions}

YSN and ARH designed this study. YSN conducted the statistical analyses and drafted the manuscript. ARH edited the manuscript. Both authors read and approved the final manuscript.

\section{Acknowledgments}

YSN is supported by a Howard Hughes Medical Institute International Student Research fellowship. The Duke Neurogenetics study is supported by Duke University. We would like to thank Annchen Knodt, Adam Gorka, Bart D. Brigidi, and Vanessa V. Sochat for their assistance in data collection and pre-processing.

Received: 23 August 2012 Accepted: 27 September 2012

Published: 14 November 2012

\section{References}

1. Stein MB, Simmons AN, Feinstein JS, Paulus MP: Increased amygdala and insula activation during emotion processing in anxiety-prone subjects. A J Psychiatry 2007, 164:318-327.

2. Glahn DC, Lovallo WR, Fox PT: Reduced amygdala activation in young adults at high risk of alcoholism: studies from the Oklahoma family health patterns project. Biol Psychiatry 2007, 61:1306-1309.

3. Sipe JC, Chiang K, Gerber AL, Beutler E, Cravatt BF: A missense mutation in human fatty acid amide hydrolase associated with problem drug use. Proc Natl Acad Sci USA 2002, 99:8394-8399.

4. Hariri AR, Gorka A, Hyde LW, Kimak M, Halder I, Ducci F, Ferrell RE, Goldman $D$, Manuck SB: Divergent effects of genetic variation in endocannabinoid signaling on human threat- and reward-related brain function. Biol Psychiatry 2009, 66:9-16.

5. Evans AH, Pavese N, Lawrence AD, Tai YF, Appel S, Doder M, Brooks DJ Lees AJ, Piccini P: Compulsive drug use linked to sensitized ventral striatal dopamine transmission. Ann Neurol 2006, 59:852-858.

6. Forbes EE, Brown SM, Kimak M, Ferrell RE, Manuck SB, Hariri AR: Genetic variation in components of dopamine neurotransmission impacts ventral striatal reactivity associated with impulsivity. Mol Psychiatry 2009, 14:60-70.

7. Sinha R: How does stress increase risk of drug abuse and relapse? Psychopharmacology (Berl) 2001, 158:343-359.

8. Rademacher DJ, Meier SE, Shi L, Ho WS, Jarrahian A, Hillard CJ: Effects of acute and repeated restraint stress on endocannabinoid content in the amygdala, ventral striatum, and medial prefrontal cortex in mice. Neuropharmacology 2008, 54:108-116.

9. Young SE, Corley RP, Stallings MC, Rhee SH, Crowley TJ, Hewitt JK: Substance use, abuse and dependence in adolescence: prevalence, symptom profiles and correlates. Drug Alcohol Depend 2002, 68:309-322.

10. Supplementary Online Methods. http://www.biolmoodanxietydisord.com/.

11. Nikolova YS, Bogdan R, Brigidi BD, Hariri AR: Ventral Striatum Reactivity to Reward and Recent Life Stress Interact to Predict Positive Affect. Biol Psychiatry 2012, 72:157-163.

12. Saunders JB, Aasland OG, Babor TF, de la Fuente JR, Grant M: Development of the Alcohol Use Disorders Identification Test (AUDIT): WHO Collaborative Project on Early Detection of Persons with Harmful Alcohol Consumption-II. Addiction 1993, 88:791-804

13. Bernstein D, Stein JA, Newcomb MD, Walker E, Pogge D, Ahluvalia T, Stokes J, Handelsman L, Medrano M, Desmond D, Zule W: Development and validation of a brief screening version of the Childhood Trauma Questionnaire. Child Abuse Negl 2002, 27:169-190.

14. Manuck SB, Brown SM, Forbes EE, Hariri AR: Temporal stability of individual differences in amygdala reactivity. A J Psychiatry 2007, 164:1613-1614.

15. Plichta MM, Schwarz AJ, Grimm O, Morgen K, Mier D, Haddad L, Gerdes AB, Sauer C, Tost H, Esslinger C, et al: Test-retest reliability of evoked BOLD signals from a cognitive-emotive fMRI test battery. Neurolmage 2012, 60:1746-1758

doi:10.1186/2045-5380-2-19

Cite this article as: Nikolova and Hariri: Neural responses to threat and reward interact to predict stress-related problem drinking: A novel protective role of the amygdala. Biology of Mood \& Anxiety Disorders 2012 $2: 19$

\section{Submit your next manuscript to BioMed Central and take full advantage of:}

- Convenient online submission

- Thorough peer review

- No space constraints or color figure charges

- Immediate publication on acceptance

- Inclusion in PubMed, CAS, Scopus and Google Scholar

- Research which is freely available for redistribution 